\title{
Successful Distal Gastrectomy for Gastric Cancer with Child-Pugh Class B Alcoholic Liver Cirrhosis
}

\author{
JUN IWABU ${ }^{1}$, TSUTOMU NAMIKAWA ${ }^{1}$, SACHI TSUDA ${ }^{1}$, HIROYUKI KITAGAWA ${ }^{1}$, \\ MICHIYA KOBAYASHI $^{2}$ and KAZUHIRO HANAZAKI ${ }^{1}$ \\ Departments of ${ }^{1}$ Surgery, and ${ }^{2}$ Human Health and Medical Sciences, \\ Kochi Medical School, Kochi, Japan
}

\begin{abstract}
Extended lymph node dissection in patients with gastric cancer with liver cirrhosis can lead to severe morbidity and mortality, especially in those with Child-Pugh class B or C cirrhosis. We, herein, report a case of advanced gastric cancer with alcoholic liver cirrhosis that was successfully treated by surgery. A 58-year-old male patient was diagnosed with gastric cancer with alcoholic liver cirrhosis Child-Pugh class B. A red blood cell transfusion was performed to treat cancer-related hemorrhage; however, the patient's hemoglobin level did not improve and distal gastrectomy with DI lymph node dissection was subsequently performed to prevent further bleeding. He was able to leave the hospital at postoperative day 16 without severe complication. Patients with liver cirrhosis can undergo distal gastrectomy with D1 lymph nodes dissection even in those with Child-Pugh class B.
\end{abstract}

Gastric cancer is one of the most commonly diagnosed tumors worldwide (1). In Japan, the gastric cancer treatment guidelines recommend gastrectomy with D2 lymph node dissection (2); however, extended lymph node dissection in patients with gastric cancer with liver cirrhosis (LC) can lead to severe postoperative complications (3-5). Thus, because LC is a major complication and terminal stage of hepatitis, the appropriate surgical strategy in patients with gastric cancer with LC remains controversial.

Several studies have examined the surgical risks in such patients $(6,7)$; however, such investigations have involved very few cases of cancer with alcoholic LC. We herein present a case of advanced gastric cancer with alcoholic LC that was successfully treated by surgery.

Correspondence to: Tsutomu Namikawa, MD, Department of Surgery, Kochi Medical School, Nankoku, Kochi 783-8505, Japan. Tel: +81 888802370, Fax: +81 888802371, e-mail: tsutomun@kochiu.ac.jp

Key Words: Gastric cancer, liver cirrhosis, gastrectomy.

\section{Case Report}

A 58-year-old male patient with LC due to long-term intake of alcohol ( $230 \mathrm{~g}$ per day) was referred to the Kochi Medical School Hospital for the treatment of gastric cancer. He had been suffering from appetite loss and leg edema for 5 months.

On admission, he had signs of anemia, ascites, and leg edema. Blood examination revealed decreased hemoglobin $(5.3 \mathrm{~g} / \mathrm{dl}$; normal range $=13.7-16.8 \mathrm{~g} / \mathrm{dl})$, increased total bilirubin $(1.8 \mathrm{mg} / \mathrm{dl}$; normal range $=0.4-1.5 \mathrm{mg} / \mathrm{dl})$, low albumin $(2.1 \mathrm{~g} / \mathrm{dl}$; normal range $=4.1-5.1 \mathrm{~g} / \mathrm{dl})$, and increased creatinine $(1.25 \mathrm{mg} / \mathrm{dl}$; normal range $=0.65-1.07 \mathrm{mg} / \mathrm{dl})$. His prothrombin time was $52.1 \%$ (normal range $=70-130 \%$ ). The staging of LC was class B, according to the Child-Pugh classification. Indocyanine green (ICG) retention rate at $15 \mathrm{~min}$ was $53.5 \%$, and esophagogastroduodenoscopy revealed a type 2 tumor with hemorrhage in the lower third of the stomach (Figure 1). Enhanced computed tomographic (CT) imaging demonstrated irregularity in the liver surface, shunting from the splenic vein to the left renal vein, increased thickness of the pylorus wall, and lymph node swelling (Figure 2). The clinical diagnosis of gastric cancer was cT4aN1M0 stage IIIA (according to the Japanese classification system).

A red blood cell transfusion was performed; however, the level of hemoglobin did not improve and distal gastrectomy with D1 lymphadenectomy and Billroth-I reconstruction were performed to prevent further bleeding of the tumor. Severe liver cirrhosis was also confirmed (Figure 3), with a small amount of ascites and hemorrhage due to portal hypertension also apparent. There were no findings of liver metastasis or peritoneal dissemination. The operative time was $240 \mathrm{~min}$, and the amount of blood loss was $460 \mathrm{ml}$. Histopathology of the tumor revealed carcinoma with enteroblastic differentiation (pT3N2M0, stage IIIA).

The patient suffered from ascites for 1 week following surgery, but his general condition was improved during that time by treatment with diuretics and fresh-frozen plasma. 


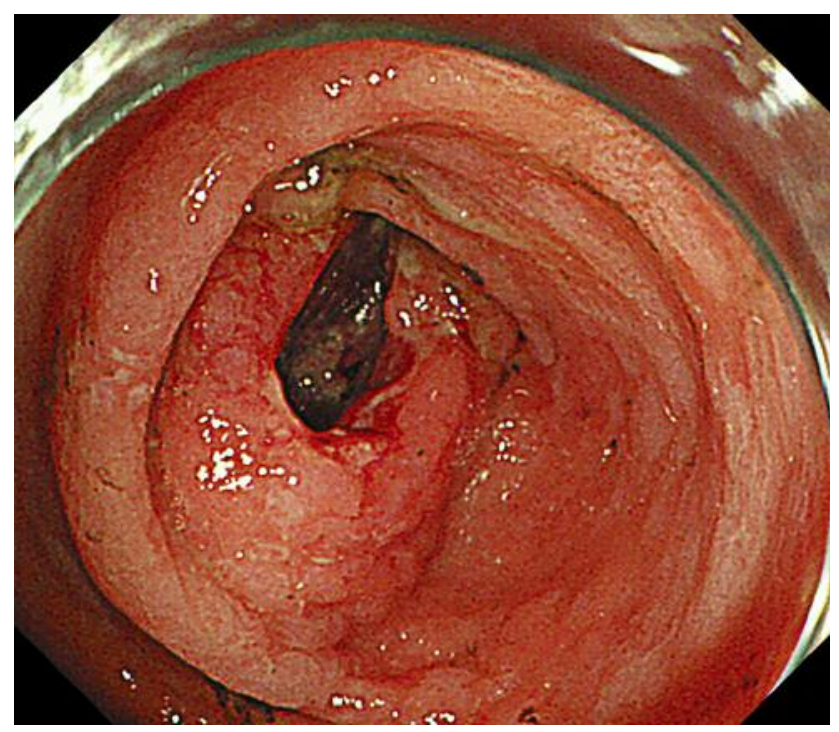

Figure 1. Upper gastrointestinal endoscopy showed type 2 advanced gastric cancer in the greater curvature of the lower gastric body.

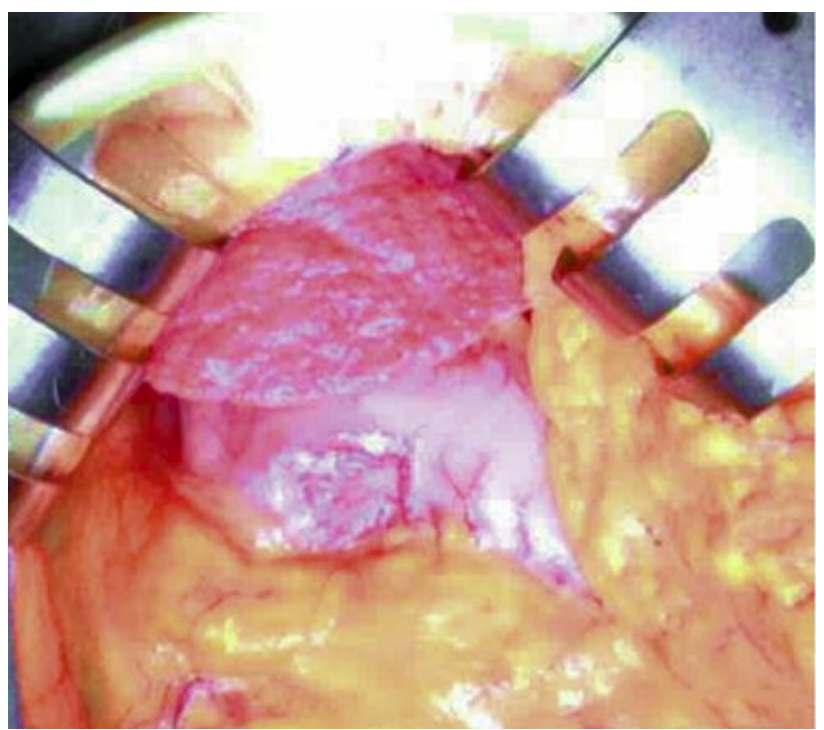

Figure 3. Liver cirrhosis was recognized during surgery.

\section{Discussion}

It is well known that abdominal surgery in patients with gastric cancer with LC is associated with high perioperative mortality, reportedly $10-85 \%(8-10)$. LC is a chronic degenerative process that occurs in a variety of diseases. Patients with LC are also at greater risk of portal hypertension, esophageal or gastric varices, and coagulopathy, with such complications leading to more blood loss, longer operations, and higher surgical risk of complications such as massive ascites.

According to previous reports, alcohol consumption of 30 $\mathrm{g}$ or more per day can induce alcoholic LC $(11,12)$. The present patient consumed approximately $230 \mathrm{~g}$ of alcohol per day over a period of time, and consequently developed severe cirrhosis as Child-Pugh class B. His pre-surgery alcohol abstinence interval was only 1 month, which was not sufficient to allow an improvement in his liver dysfunction.

Despite the high risk of adverse effects, little is known about suitable surgical methods for patients with gastric cancer with LC. According to one retrospective study, D2 lymph node dissection should be avoided in patients with Child-Pugh class B cirrhosis, based on the fact that three out of four patients with grade B cirrhosis who underwent D2 lymph node dissection developed massive ascites postoperatively (6). Another study reported that D2 lymph node dissection produced no improvement in survival length over D1 lymph node dissection for patients with Child-Pugh class B (7). In contrast, another retrospective study reported 
that extended lymph node dissection is safe for those with Child-Pugh class A cirrhosis (13). Together these findings suggest that extended, lymph node dissection should be avoided in patients with Child-Pugh class B cirrhosis.

In the present case, we performed distal gastrectomy to prevent bleeding from gastric cancer, with only a DI lymph node dissection performed to prevent postoperative liver dysfunction. During the perioperative period, fresh-frozen plasma and a diuretic were used to prevent intractable ascites. Malnutrition is also a potential clinical problem in patients with alcoholic LC (14), and because this patient lacked a proper diet including proteins and vitamins, we also administered an elemental diet including branched-chain amino acids from admission to maintain a good postoperative nutritional status. These findings indicate that appropriate and intensive postoperative management, such as an elementary diet and diuretics, is important to achieve satisfactory outcomes for patients with patients with LC who undergo surgery for gastric cancer.

\section{Conclusion}

We report a case of successful surgery with D1 lymph node dissection for gastric cancer with Child-Pugh class B alcoholic LC. D1 lymph node dissection is adequate for patients with Child-Pugh class B to prevent perioperative complications.

\section{References}

1 Jemal A, Bray F, Center MM, Ferlay J, Ward E and Forman D: Global cancer statistics. CA Cancer J Clin 61(2): 69-90, 2011.

2 Japanese Gastric Cancer Association: Japanese gastric cancer treatment guidelines 2014 (ver.4). Gastric Cancer 20(1): 1-19, 2017.

3 Takeda J, Hashimoto K, Tanaka T, Koufuji K and Kakegawa T: Review of operative indication and prognosis in gastric cancer with hepatic cirrhosis. Hepatogastroenterology 39(5): 433-436, 1992.
4 Isozaki H, Okajima K, Ichinona T, Fujii K, Nomura E and Izumi N: Surgery for gastric cancer in patients with cirrhosis. Surg Today 27(1): 17-21, 1997.

5 Kumagai K: Intractable ascites following surgery for gastric carcinoma. Dig Surg 15(3): 236-240, 1998.

6 Jang HJ, Kim JH, Song HH, Woo KH, Kim M, Kae SH, Lee J, Cho JW, Kang JH, Lee SI, Gong SJ, Lee JA and Zang DY: Clinical outcomes of patients with liver cirrhosis who underwent curative surgery for gastric cancer: a retrospective multi-center study. Dig Dis Sci 53(2): 399-404, 2008.

7 Guo F, Ma S, Yang S, Dong Y, Luo F and Wang Z: Surgical strategy for gastric cancer patients with liver cirrhosis: a retrospective cohort study. Int J Surg 12(8): 810-814, 2014.

8 Mansour A, Watson W, Shayani V and Pickleman J: Abdominal operations in patients with cirrhosis: still a major surgical challenge. Surgery 122(4): 730-736, 1997.

9 Northup PG, Wanamaker RC, Lee VD, Adams RB and Berg CL: Model for end-stage liver disease (MELD) predicts nontransplant surgical mortality in patients with cirrhosis. Ann Surg 242(2): 244-251, 2005.

10 Neeff HP, Streule GC, Drognitz O, Tittelbach-Helmrich D and Spangenberg HC, Hopt UT and Makowiec F: Early mortality and long-term survival after abdominal surgery in patient with liver cirrhosis. Surgery 155(4): 623-632, 2014.

11 Bruha R, Dvorak K and Petrtyl J: Alcohol liver disease. World J Hepatol 4(3): 81-90, 2012.

12 Bellentani S and Tiribelli C: The spectrum of liver disease in the general population: lesson from the Dionysos study. J Hepatol 35(4): 531-537, 2001.

13 Ryu KW, Lee JH, Kim YW, Park JW and Bae JM: Management of ascites after radical surgery in gastric cancer patients with liver cirrhosis and minimal hepatic dysfunction. World J Surg 29(5): 653-656, 2005.

14 Toshikuni N, Arisawa T and Tsutsumi M: Nutrition and exercise in the management of liver cirrhosis. World J Gastroenterol 20(23): 7286-7297, 2014.
Received February 27, 2018

Revised March 14, 2018

Accepted March 20, 2018 